\title{
Quantum Spectrum of Three Anyons in an Oscillator Potential
}

\author{
M. V. N. Murthy, ${ }^{(a)}$ J. Law, ${ }^{(b)}$ M. Brack, ${ }^{(c)}$ and R. K. Bhaduri \\ Physics Department, McMaster University, Hamilton, Ontario, Canada L8S 4MI
}

(Received 10 June 1991)

\begin{abstract}
The low-lying quantum states of three anyons confined in a harmonic-oscillator well are obtained numerically. The interpolation of these states between the limits of noninteracting bosons to noninteracting fermions in the well is established. Particular attention is paid to the transformation of the fermionic ground state to an excited Bosonic state, and its overlap with Laughlin-Wu-type correlated states.
\end{abstract}

PACS numbers: 03.65.Ge, 74.65.+n

In two space dimensions, particles may obey fractional statistics and have multiple-valued wave functions [1-4]. The quantum mechanics of such particles may be studied by considering them to be bosons (or fermions) having single-valued wave functions, but interacting with longrange Aharonov-Bohm-type vector potentials. Whereas many-anyon systems have been studied in both the single-particle [5] as well as collective hydrodynamic [6] approaches, not much attention has been paid to solving the few-anyon problem. The quantum spectrum of two anyons in a harmonic oscillator [1] or on a circle [7] was solved early, and used to evaluate the second virial coefficient of an anyon gas [7,8]. The quantum problem of three electrons in a strong magnetic field, occupying the same Landau level, was examined in detail by Laughlin [9], which led him to propose the Jastrow-type correlated variational many-body wave function that is so successful in the explanation of the fractional quantum Hall effect [10]. Such a Laughlin state was shown [11] to be the exact nondegenerate ground state of a many-particle system in a uniform magnetic field, interacting with a repulsive interaction of vanishing range. Numerical eigenvalues of such few-particle systems with various filling factors were studied in this connection. It should also be noted that numerical studies of few-semion systems have been made on a square lattice with periodic boundary conditions in one direction [12] and on a spherical surface [13] (to eliminate edge effects) with a view to study semion pairing in the ground state.

The problem of a few-anyon system in the absence of an external magnetic field and confined in a potential well is rather different, however, and is worth studying to examine the interpolation of these states between the limits of Bose and Fermi results. The three-anyon problem in a harmonic potential was first studied in this connection by $\mathrm{Wu}$ [14]. He eliminated the long-range vector potential by a gauge transformation, and proposed a multiplevalued correlated state of Laughlin type that interpolated linearly between the Bose and Fermi limits. The bosonic ground state transmutes in this manner to an excited fermionic state carrying three units (in $\hbar$ ) of angular momentum. The behavior of the fermionic ground state, together with a whole class of other excited states, is more complicated, however, and throws some light on the quantum mechanics of many-anyon systems. The pri- mary aim of this paper is to study the behavior of these low-lying states as a function of the strength of the statistical interaction. Such a study has also been made by Sporre, Verbaarschot, and Zahed [15] using a different method. Khare and McCabe [16] have recently studied the behavior of the fermionic ground state perturbatively in this connection. At the end, we shall give a simple physical interpretation of our numerical work.

For this purpose, we express the three-anyon Hamiltonian in Jacobi coordinates and eliminate the center-ofmass part. A general method of constructing a completely symmetric (or antisymmetric) basis is then briefly outlined, and the Hamiltonian diagonalized in such a basis. The Lagrangian for the three-anyon problem is given by

$$
\begin{aligned}
L= & \frac{1}{2} m\left(\dot{\mathbf{r}}_{1}^{2}+\dot{\mathbf{r}}_{2}^{2}+\dot{\mathbf{r}}_{3}^{2}\right)+h \alpha \sum_{j>i=1}^{3} \dot{\theta}_{i j} \\
& -\frac{1}{2} m \omega^{2}\left(r_{1}^{2}+r_{2}^{2}+r_{3}^{2}\right),
\end{aligned}
$$

where $\mathbf{r}_{i}$ denote the particle coordinates. The second term is the statistical interaction whose strength is given by $\alpha$ (in the range $0 \leq \alpha \leq 1)$ and $\theta_{i j}=\tan ^{-1}\left[\left(y_{i}-y_{j}\right) /\right.$ $\left(x_{i}-x_{j}\right)$ ]. The corresponding Hamiltonian is given by

$$
\begin{aligned}
H= & \frac{1}{2 m} \sum_{i=1}^{3} \mathbf{p}_{i}^{2}+\frac{1}{2} m \omega^{2} \sum_{i=1}^{3} \mathbf{r}_{i}^{2}-\frac{\alpha}{m} \sum_{j>i=1}^{3} \frac{\hat{l}_{i j}}{r_{i j}^{2}} \\
& +\frac{\alpha^{2}}{2 m} \sum_{i \neq j \neq k)=1}^{3} \frac{\left(\mathbf{r}_{i j}+\mathbf{r}_{i k}\right)^{2}}{r_{i j}^{2} r_{i k}^{2}},
\end{aligned}
$$

where $\hat{l}_{i j}=\left(\mathbf{r}_{i}-\mathbf{r}_{j}\right) \times\left(\mathbf{p}_{i}-\mathbf{p}_{j}\right)$.

After eliminating the center of mass, one is left with two internal coordinates which may be chosen to be $\boldsymbol{\rho}=(1 / \sqrt{2})\left(\mathbf{r}_{1}-\mathbf{r}_{2}\right)$ and $\lambda=(1 / \sqrt{6})\left(\mathbf{r}_{1}+\mathbf{r}_{2}-2 \mathbf{r}_{3}\right)$. Since the Hamiltonian is symmetric, while computing the matrix elements of $\boldsymbol{H}$ with a symmetric (or antisymmetric) set of states, it is sufficient to consider only one of the three terms involving the statistical interaction multiplied by 3 . The Hamiltonian in relative coordinates, after eliminating the center of mass, is

$$
\begin{aligned}
H_{\mathrm{rel}}= & \frac{1}{2 m}\left(\mathbf{p}_{\rho}^{2}+\mathbf{p}_{\lambda}^{2}\right)+\frac{1}{2} m \omega^{2}\left(\rho^{2}+\lambda^{2}\right) \\
& +\frac{3}{m \rho^{2}}\left[-\alpha \hat{l}_{\rho}+\frac{3}{4} \alpha^{2} \frac{(\sqrt{3} \rho+\lambda)^{2}}{(\rho+\sqrt{3} \lambda)^{2}}\right] .
\end{aligned}
$$


We now briefly discuss the method of constructing the basis. We choose as our basis the eigenstates of the harmonic-oscillator Hamiltonian with three particles. We first note that all such states may be expressed as finite inhomogeneous polynomials in $\rho$ and $\lambda$ apart from the Gaussian factor $\exp \left[-\frac{1}{2} m \omega\left(\rho^{2}+\lambda^{2}\right)\right]$, which is completely symmetric under permutation of the $r_{1}$ 's. Thus the symmetry of the polynomial completely determines the symmetry of the state. Below we indicate our method of constructing homogeneous polynomials (in $\rho$ and $\lambda$ ) of definite permutation symmetry.

If $\boldsymbol{P}_{i j}$ denotes a permutation of $i, j$ particle coordinates, then we have

$$
\begin{aligned}
& P_{12} \rho_{ \pm}=-\rho_{ \pm}, \quad P_{12} \lambda_{ \pm}=+\lambda_{ \pm}, \\
& P_{23} \rho_{ \pm}=\frac{1}{2}\left(\rho_{ \pm}+\sqrt{3} \lambda_{ \pm}\right), \\
& P_{23} \lambda_{ \pm}=\frac{1}{2}\left(\sqrt{3} \rho_{ \pm}-\lambda_{ \pm}\right),
\end{aligned}
$$

where $\rho_{ \pm}=\rho_{x} \pm i \rho_{y}$ and similarly for $\lambda_{ \pm}$. Notice that $\rho_{ \pm}$and $\lambda_{ \pm}$are polynomials of degree 1 with angular momentum $l_{\rho}$ and $l_{\lambda}= \pm 1$. In general, if $\phi_{\rho}^{N_{1} L_{1}}$ denotes a polynomial of degree $N_{1}$ and angular momentum $L_{1}$, which transforms like $\rho$ under permutations and $\phi_{\lambda}^{N_{2} L_{2}}$ a polynomial of degree $N_{2}$ and angular momentum $L_{2}$ which transforms like $\lambda$, then we have the following classification of polynomials constructed out of $\phi_{\rho}$ and $\phi_{\lambda}$ :

$$
\begin{aligned}
& \phi_{S}^{N, L}=\phi_{\rho}^{N_{1} L_{1}} \phi_{\rho}^{N_{2} L_{2}}+\phi_{\lambda}^{N_{1} L_{1}} \phi_{\lambda}^{N_{2} L_{2}} \\
& \phi_{\rho}^{N, L}=\phi_{\rho}^{N_{1} L_{1}} \phi_{\lambda}^{N_{2} L_{2}}+\phi_{\rho}^{N_{2} L_{2}} \phi_{\lambda}^{N_{1} L_{1}} \quad \text { (mixed symmetry } \rho \text { type), } \\
& \phi_{\lambda}^{N, L}=\phi_{\rho}^{N_{1} L_{1}} \phi_{\rho}^{N_{2} L_{2}}-\phi_{\lambda}^{N_{1} L_{1}} \phi_{\lambda}^{N_{2} L_{2}} \quad \text { (mixed symmetry } \lambda \text { type), } \\
& \phi_{A}^{N, L}=\phi_{\rho}^{N_{1} L_{1}} \phi_{\lambda}^{N_{2} L_{2}}-\phi_{\rho}^{N_{2} L_{2}} \phi_{\lambda}^{N_{1} L_{1}} \quad \text { (antisymmetric), }
\end{aligned}
$$

where $N=N_{1}+N_{2}, L=L_{1}+L_{2}$. Equations (5) provide a recursive algorithm for constructing all possible states of a given $N$ and $L$ starting from the elementary $\phi$ 's, $\phi_{\rho}^{1, \pm 1}=\rho_{ \pm}$and $\phi_{\lambda}^{1, \pm 1}=\lambda_{ \pm}$.

It is easy to see that there are no symmetric or antisymmetric polynomials for $N=1$, while the first antisymmetric polynomial occurs at $N=2, \phi_{A}^{2,0}=\rho_{+} \lambda_{-}$ $-\rho-\lambda_{+}$. This indeed corresponds to the fermionic ground state which occurs at energy $4 \hbar \omega$ for $\alpha=0$ in Eq. (3). We should, however, caution that even though Eq. (5) provides a global algorithm to construct polynomials of all possible symmetries under the permutation of three particles, not all the polynomials for a given $N, L$ and symmetry constructed from $N_{1}$ and $N_{2}$ are distinct. Construction of such irreducible sets will be discussed elsewhere. However, for the $L=0$ symmetric states, the following combination completely exhausts all polynomials (see, for example, Ref. [17]):

$$
\phi_{S}^{N, 0}=S_{2}^{i} S_{4}^{j} S_{6}^{k}, \quad N=2 i+4 j+6 k,
$$

where

$$
\begin{aligned}
& S_{2}=\rho^{2}+\lambda^{2}, \quad S_{4}=(2 \rho \cdot \lambda)^{2}+\left(\rho^{2}-\lambda^{2}\right)^{2}, \\
& S_{6}=\left(\rho^{2}-\lambda^{2}\right)\left[\left(\rho^{2}-\lambda^{2}\right)^{2}-3(2 \rho \cdot \lambda)^{2}\right]
\end{aligned}
$$

are each symmetric under permutations. Correspondingly the antisymmetric $L=0$ polynomials may be written as $\phi_{A}^{N, 0}=\phi_{A}^{2,0} \phi_{S}^{N-2,0}$. Unlike $\phi_{S}^{N, 0}$, this does not exhaust all the antisymmetric polynomials, and the lowest of these at $N=6$ (with energy $8 \hbar \omega$ ) has the structure

$$
\phi_{A}^{6.0}=(2 \rho \cdot \lambda)\left\{3\left(\rho^{2}-\lambda^{2}\right)^{2}-[2(\rho \cdot \lambda)]^{2}\right\} .
$$

A new class of antisymmetric states may then be generated starting at $8 \hbar \omega$, denoted by $\tilde{\phi}_{A}^{N, 0}=\phi_{A}^{6,0} \phi_{S}^{N-6,0}$. In our calculations in this Letter, these states were not included.

Instead of constructing the eigenstates of the oscillator
Hamiltonian, we propose to find the eigenvalues of $H_{\mathrm{rel}}$ in the nonorthogonal basis provided by the set of all symmetric (antisymmetric) polynomials of a given $L$. However, since the bosonic states do not necessarily vanish at the origin, the centrifugal terms in $H_{\text {rel }}$ cause the energy to diverge. Following $\mathrm{Wu}$, we therefore introduce the Jastrow-type pair correlations and define

$$
\psi S, L, L(\rho, \lambda)=\prod_{i<j}\left|\mathbf{r}_{i}-\mathbf{r}_{j}\right|^{\alpha} \phi S,{ }_{A}^{N} e^{-m \omega^{2}\left(\rho^{2}+\lambda^{2}\right) / 2},
$$

where $\alpha$ is the strength of the anyonic interaction. The correlation factor is symmetric and does not alter the symmetry of the state. For $\alpha \neq 0$, this factor introduces zeros in the wave function necessary to avoid divergence. In addition, we find that the overlap defined as $O^{N N^{\prime}}$ $=\left\langle\psi_{S}^{N^{\prime}, L^{\prime}}\left|\prod_{i<j} e^{i \theta_{i j}}\right| \psi_{A}^{N, L}\right\rangle$ is appreciable only when $N^{\prime}$ is close to $N$, at least for the low-lying states.

It is interesting to note that in some special cases $O^{N N^{\prime}}=1$, indicating a one-to-one correspondence between some states. For example, the $L=0$ bosonic ground state has structure $\prod_{i<j}\left|\mathbf{r}_{i}-\mathbf{r}_{j}\right|^{\alpha} e^{-m \omega^{2}\left(\rho^{2}+\lambda^{2}\right) / 2}$ with energy $2 \hbar \omega$ at $\alpha=0$. At $\alpha=1$, the corresponding anyon wave function is given by

$$
\prod_{i<j} e^{i \theta_{i j}}\left|\mathbf{r}_{i}-\mathbf{r}_{j}\right| e^{-m \omega^{2}\left(\rho^{2}+\lambda^{2}\right) / 2}=\phi_{A}^{3,3} e^{-m \omega^{2}\left(\rho^{2}+\lambda^{2}\right) / 2},
$$

which is in fact the lowest fermionic oscillator eigenstate with $L=3, N=3$, and energy $5 \hbar \omega$. Indeed, as we shall see later, in all such cases where there exists a one-to-one correspondence the interpolation in energy is linear. These are precisely the states singled out by $\mathrm{Wu}$. The $N=2$ fermionic ground state, however, has overlaps $O^{N N^{\prime}}$ with bosonic $L=3$ states with $N=3(65 \%)$ and $N=5$ $(35 \%)$. The interpolation in this and similar cases is nonlinear.

The result of diagonalizing the anyonic Hamiltonian 


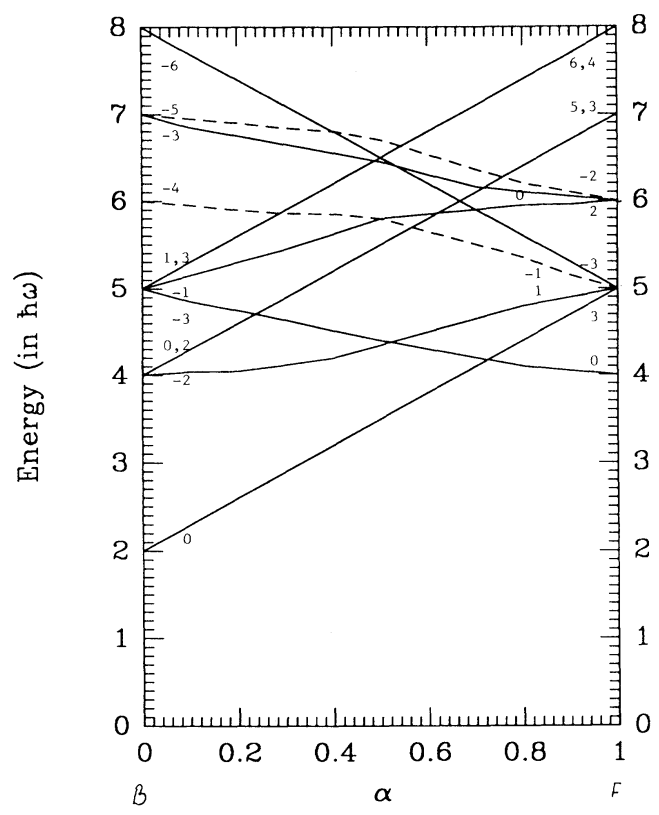

FIG. 1. The low-lying energy levels of the Hamiltonian [Eq. (3)] are displayed as a function of $\alpha$. The angular momenta of the states are labeled at the ends. At the left end, $\alpha=0$ corresponds to the relative energy of three noninteracting bosons in an oscillator while at the right extreme $\alpha=1$ is for three noninteracting fermions in the oscillator. The dashed curves indicate that the diagonalization is done from the Fermi end to $\alpha=\frac{1}{2}$, and the extrapolation to the bosonic end is schematic. All states up to $5 \hbar \omega$ are shown. At $6 \hbar \omega$, there are only three states at the fermionic end (all shown) and six at the bosonic end (only one shown). The number of states increases rapidly from $7 \hbar \omega$.

given by Eq. (3) for various values of $\alpha$ (in the range $0<\alpha<1)$ is displayed in Fig. 1. For economy of the basis, this range is spanned by starting from the Bose as well as Fermi ends separately and matching the eigenvalues at $\alpha=\frac{1}{2}$, especially when the variation is nonlinear. Typically, for a given $L$, we took about ten states in a basis. Adding a few more changed the lowest eigenvalue only within $1 \%$. This does not guarantee, however, that the energy would not be lowered more if the basis were increased by many more states. A more exhaustive study of convergence will be presented elsewhere [18], where a more detailed exposition of the formalism will also be given.

In Fig. 1, the interpolation pattern of states with $|L| \leq 3$ and only up to $6 \hbar \omega$ are shown. It will be seen from the spectrum that the time-reversed states $(1,-1)$ are split in energy by the anyonic interaction. In our convention, $L$ increases by $3 \hbar$ in going from the bosonic to the fermionic end. Those states that interpolate linearly change in energy by $3 \hbar \omega$ in this range, while the nonlinear ones change in energy only by $\hbar \omega$. These nonlinearly interpolating states suffer large mixing of the basis states for nonzero $\alpha$, while the linear ones remain almost pure. The large number of level crossings results in the bunching of quantum states for $\alpha=\frac{1}{2}$ and $\frac{2}{3}$ and also possibly at $\frac{1}{3}$.

Finally, a simple physical interpretation of the linear and nonlinear interpolating states in Fig. 1 may be given. Consider the bosonic ground state at $\alpha=0$ where all three particles are in the $1 s$ state. (In Fig. 1, the energy along the $y$ axis is shown after the center-of-mass energy of $1 \hbar \omega$ is subtracted off.) This state goes over to a noninteracting fermionic state with relative energy $5 \hbar \omega$, with one particle each in $1 s, 1 p$, and $1 d$ states, with total $L=3$. There is only one such state with $L=3$ at $5 \hbar \omega$. This one-to-one correspondence yields linear behavior in interpolation. On the other hand, the fermionic ground state with $L=0$ (at $E=4 \hbar \omega$ ) has one particle in $1 s$ and two in 1p. This state interpolates on the bosonic side to a state with $E=5 \hbar \omega$ and $L=-3$ that is a linear combination of the configurations $[1 s, 1 p, 1 d],\left[(1 p)^{3}\right]$, and $\left[(1 s)^{2}, 1 f\right]$. This more complicated structure seems to lead to the nonlinear behavior in Fig. 1, and the anyonic energy may only be found numerically for such states.

We wish to thank Professor Peter Eglestaff and the SNO group at the University of Guelph for use of their computing facilities. The authors are very thankful to Professor Gerry Brown and Professor A. Khare for bringing to our notice Refs. [15] and [16], respectively. This research was supported by grants from NSERC of Canada. J.L., M.V.N.M., and M.B. acknowledge the hospitality of the Physics Department, McMaster University.

(a) Permanent address: Institute of Mathematical Sciences, Madras, India.

(b) Permanent address: Department of Physics, University of Guelph, Ontario, Canada.

(c) Permanent address: Institute of Theoretical Physics, University of Regensburg, D8400 Regensburg, Germany.

[1] J. M. Leinaas and J. Myrheim, Nuovo Cimento 37B, 1 (1977).

[2] F. Wilczek, Phys. Rev. Lett. 48, 1144 (1982); 49, 957 (1982).

[3] F. Wilczek and A. Zee, Phys. Rev. Lett. 51, 2250 (1983).

[4] For some recent reviews, see R. Mackenzie and F. Wilczek, Int. J. Mod. Phys. A 12, 2827 (1988); G. S. Canright and M. D. Johnson, Comments Condens. Matter Phys. 15, 77 (1990).

[5] A. L. Fetter, C. B. Hanna, and R. B. Laughlin, Phys. Rev. B 39, 9679 (1989).

[6] X. G. Wen and A. Zee, Phys. Rev. B 41, 240 (1990).

[7] D. P. Arovas, R. Schrieffer, F. Wilczek, and A. Zee, Nucl. Phys. B251 [FS13], 117 (1985).

[8] A. Comtet, Y. Georgelin, and S. Ouvry, J. Phys. A 22, 3917 (1989).

[9] R. B. Laughlin, Phys. Rev. B 27, 3383 (1983).

[10] R. B. Laughlin, Phys. Rev. Lett. 50, 1395 (1983).

[11] S. A. Trugman and S. Kivelson, Phys. Rev. B 31, 5280 (1985). 
[12] G. S. Canright, S. M. Girvin, and A. Brass, Phys. Rev. Lett. 63, 2295 (1989).

[13] Weiking Wu, C. Kallin, and A. Brass, Phys. Rev. B 42, 2222 (1990).

[14] Yong-Shi Wu, Phys. Rev. Lett. 53, 111 (1984).

[15] M. Sporre, J. J. M. Verbaarschot, and I. Zahed, preced- ing Letter, Phys. Rev. Lett. 67, 1813 (1991).

[16] Avinash Khare and John McCabe, Orsay Report No. IPNO/TH 91-48 (to be published).

[17] G. Karl and E. Obryk, Nucl. Phys. B8, 809 (1968).

[18] M. V. N. Murthy, J. Law, M. Brack, and R. K. Bhaduri (to be published). 